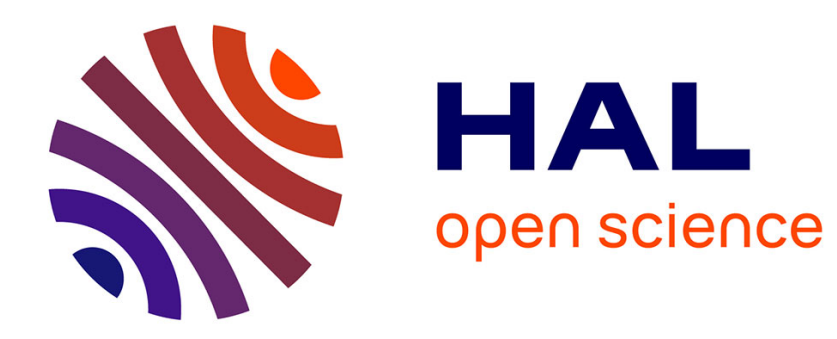

\title{
La froideur du regard impassible des États Ariel Colonomos
}

\section{To cite this version:}

Ariel Colonomos. La froideur du regard impassible des États. Communications, 2004, 75, pp.75-90. hal-01019669

\section{HAL Id: hal-01019669 \\ https://hal-sciencespo.archives-ouvertes.fr/hal-01019669}

Submitted on 7 Jul 2014

HAL is a multi-disciplinary open access archive for the deposit and dissemination of scientific research documents, whether they are published or not. The documents may come from teaching and research institutions in France or abroad, or from public or private research centers.
L'archive ouverte pluridisciplinaire HAL, est destinée au dépôt et à la diffusion de documents scientifiques de niveau recherche, publiés ou non, émanant des établissements d'enseignement et de recherche français ou étrangers, des laboratoires publics ou privés. 


\title{
? Ariel Colonomos
}

\section{La froideur du regard impassible des États}

In: Communications, 75, 2004. pp. 75-90.

\begin{abstract}
The esthetics of power have anticipated and influenced the construction of a rational and amoral theory of international relations called realism. In this paper, the analysis of several portraits of statesmen and diplomats focuses on the representation of the eyes of power. Three major characters have to be taken into account in this process : the painter, the diplomat and the scholar. They are the true protagonists of the emergence of this rationality that has played a great role, for better and most often for worse, in international politics.
\end{abstract}

\section{Résumé}

À travers l'analyse de certains portraits de grands dignitaires de l'État, ce texte explore les rapports entre une esthétique de la représentation du pouvoir et une rationalité d'une science réaliste des relations internationales fondée sur la distanciation amorale. Dans l'incarnation de l'État par les grands, cette esthétique du regard communique avec cette science de la vision. Notre analyse montre que le trait précède la science et qu'à travers le travail du peintre l'intuition du regard de l'État préfigure la construction d'un savoir froid et objectivant. Les correspondances entre ces trois regards - le peintre, le diplomate et le savant — sont à l'origine d'une énigme dont la raison du politique est le cœur.

Citer ce document / Cite this document :

Colonomos Ariel. La froideur du regard impassible des États. In: Communications, 75, 2004. pp. 75-90.

doi : $10.3406 /$ comm.2004.2144

http://www.persee.fr/web/revues/home/prescript/article/comm_0588-8018_2004_num_75_1_2144 


\section{Ariel Colonomos}

\section{La froideur du regard impassible des Etats}

L'Etat, c'est le plus froid de tous les monstres froids. Il ment froidement; et voici le mensonge qui rampe de sa bouche : "Moi, l'État, je suis le Peuple. "

Friedrich Nietzsche ${ }^{1}$

Traditionnellement, l'État est associé à une figure de la raison. Une telle disposition suppose, pour le gouvernant et ses auxiliaires, impassibilité et maîtrise de soi. Ces caractéristiques physiques et cette nature s'incarnent dans les postures des grands, tout particulièrement lorsque le pouvoir se donne à voir publiquement. À ce titre, la peinture puis, d'une manière plus générale, l'image nous renseignent à la fois sur ce désir de paraître conforme à l'impassibilité et sur la volonté du pouvoir d'être associé à la froideur.

Cette disposition est le reflet d'une raison du politique fondée sur l'anticipation et le calcul, des formes de l'esprit qui nécessitent le recul et la distance dont la froideur, la retenue et l'impassibilité seraient les garants. La maîtrise de l'émotion a valeur de science dans un tel contexte et celle-ci se donne pour vocation d'occuper toutes les sphères du politique.

Il est un domaine où cette froideur calculatrice a été érigée en modèle avec une attention plus particulière : l'art de gouverner dans les relations internationales. Cet art procéderait d'un talent, l'anticipation du lointain, une vision du large que permet l'auxiliaire de la carte et du binocle ou de la longue-vue (en italien : il cannocchiàle, la canne doublée de lunettes et d'un œeil). Le chef d'État lorsqu'il s'occupe des affaires étrangères, l'ambassadeur, le conseiller du Prince en matière de diplomatie et de stratégie : ces différentes figures ont fait de la froideur et du calcul les vertus cardinales de l'exercice de leurs fonctions. Limage traduit cet art de gouverner et la peinture du $\mathrm{XVI}^{\mathrm{e}}$ et du $\mathrm{XVII}^{\mathrm{e}}$ siècle nous renseigne sur la disposition des grands à vouloir endosser les habits du pouvoir en se parant du masque de l'impénétrable.

L'histoire de la peinture à l'âge moderne illustre cette volonté de paraître qui marque de son empreinte les tableaux représentant les dignitaires de 


\section{Ariel Colonomos}

l'État. Cette plastique révèle une politique qui, elle-même, sous-tend un savoir ${ }^{2}$. Le regard froid et distancié de l'homme qui s'occupe du domaine le plus vaste qui soit - le monde - est le signe d'une science auxiliaire du pouvoir.

Interrogeons sa genèse en la rapportant à la nature du pouvoir qu'elle vient seconder. En tant que discipline, les relations internationales sont profondément marquées par une école qui prend le nom de « réalisme ». Le réalisme affiche sa volonté de faire science dans l'art du politique ${ }^{3}$. Le réalisme est la théorie de l'art de gouverner suivant le mode de la raison distanciée; il a connu son heure de gloire au moment de la guerre froide sans qu'aucun rival ait été en mesure de le concurrencer dans l'explication du fait international. Par son nom, le réalisme, qui allie science des régularités et connaissance des particularités géographiques, suppose d'emblée la métaphore du regard. Conseillé par un savant dans l'art de gouverner, l'homme d'État apprend à voir le monde tel qu'il est, désireux qu'il est de se prémunir en tout premier lieu contre les pièges d'une vision prisonnière de l'aveuglement d'un idéalisme naîf. Le réaliste fait de la distanciation amorale une vertu de la découverte scientifique, qui oriente une juste définition de la puissance. Cette intelligence de l'homme d'État l'engage à prendre du recul en tenant à distance, par la raison, le lointain.

L'image du pouvoir, l'art de gouverner et la théorie de la gouvernance sont trois différents niveaux de cette réflexion sur le juste regard du pouvoir. Il est avant tout crucial de comprendre les correspondances entre ces trois dimensions en mettant l'accent sur le lien entre une esthétique et une épistémologie. Cette démarche mène à une analyse qui éclaire l'anticipation du trait et de la représentation sur la théorie et les concepts. D'emblée on prend conscience des relations entre trois personnages dont les regards se réfractent les uns sur les autres : tout d'abord ceux du peintre et de son modèle, qui de concert représentent la grandeur par la froideur distanciée, puis celui du savant conseiller, qui fait œuvre de science par la distanciation et l'objectivation du réel.

\section{Le réalisme kissingérien au miroir du cardinal de Richelieu.}

La diplomatie a une icône : le cardinal de Richelieu représenté dans les tableaux de Philippe de Champaigne. Le Cardinal avait la charge des affaires de l'État et tenait les rênes de la politique étrangère du royaume de France au moment de la guerre de Trente Ans. Par-delà le XVII ${ }^{e}$ siècle, Richelieu désigne la place d'un moment religieux crucial dans l'histoire des relations entre États. 


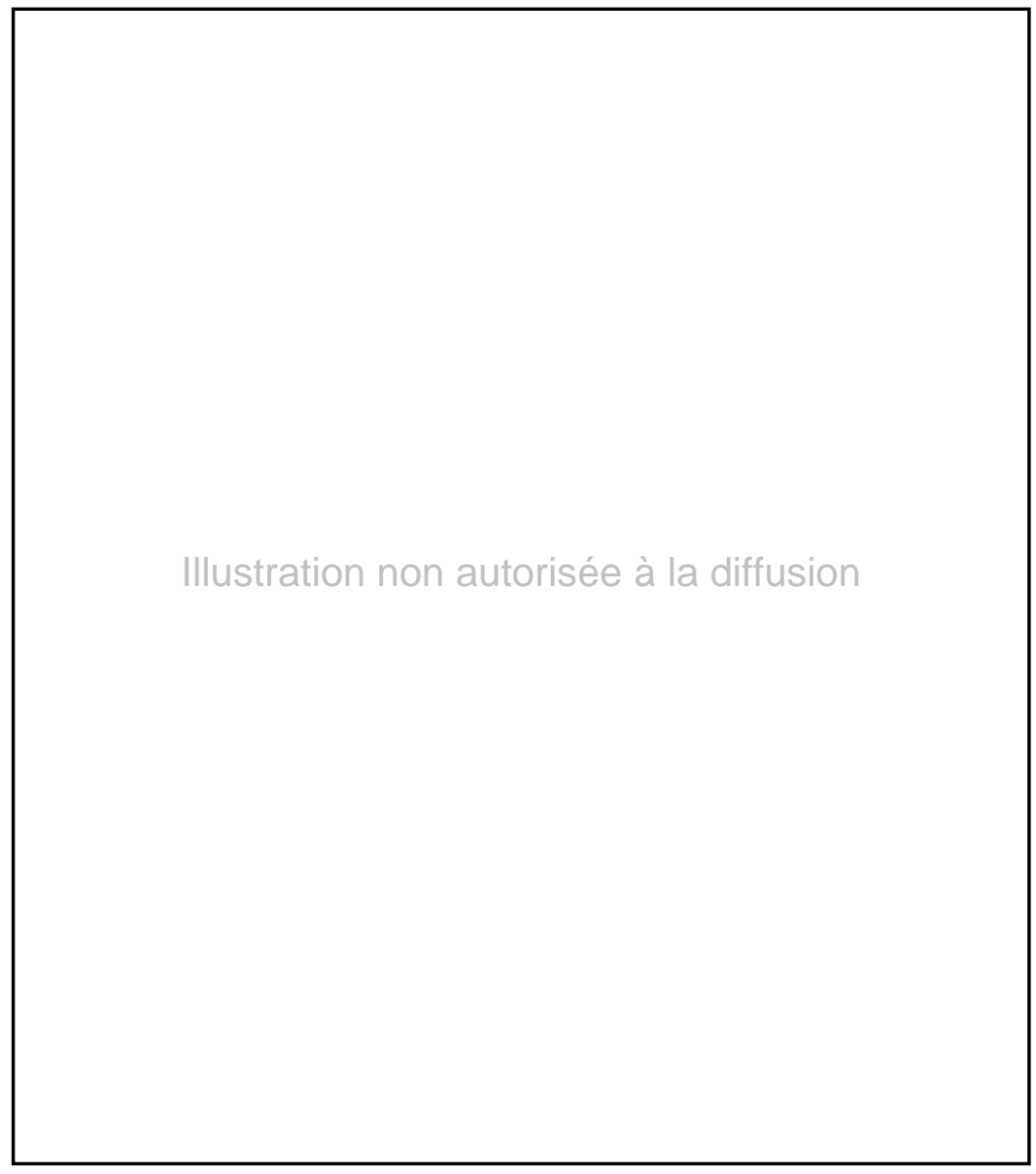

Portrait d'Armand Jean du Plessis, cardinal de Richelieu, par Philippe de Champaigne.

(C) Bridgeman-Giraudon.)

« Diplomacy, in the sense of ordered conduct of relations between one group of human beings and another group alien to themselves, is far older than history. The theorists of the l6th century contended that the first diplomatists were angels, in that they served as "angeloi" or messengers between heaven and earth" (Harold Nicolson, Diplomacy).

Dans sa politique intérieure, Richelieu n'eut de cesse de combattre l'avancée protestante, notamment en favorisant les mouvements les plus zélés de la Contre-Réforme, comme les Jésuites. En matière de politique étrangère, il s'appliqua méticuleusement à étendre la puissance du royaume de France. La maximisation de cet objectif supposait de nuire aux intérêts de l'Église catholique romaine; en effet, l'intérêt d'un État comme la France réclamait que le Saint Empire romain germanique des Habsbourg fût diminué. En conséquence, le Cardinal conclut des pactes avec des royaumes protestants du Nord pour ébranler l'unité du Saint Empire.

Le calcul froid et distancié de Richelieu dans sa conduite de la guerre l'a fait entrer dans l'histoire des relations internationales, où, traditionnellement, il est associé au cynisme - une qualité morale qui désigne dans certaines théories du politique un atout indispensable dans l'exercice du pouvoir. 


\section{Ariel Colonomos}

Les tableaux de Champaigne représentent le Cardinal sous les traits d'un homme au regard impassible, venant signifier une profonde distance vis-à-vis du monde qui l'entoure. Cette distance avec le réel est une arme qui permet à Richelieu de donner une assise à une décision stratégique audacieuse. Elle le rend d'autant plus imprévisible, tout en attirant sur lui le regard de ses partenaires comme de ses adversaires. L'impassibilité institue de facto. le stratège ${ }^{4}$.

Triple portrait de la tête de Richelieu, par Philippe de Champaigne (1642).

(@) Bridgeman-Giraudon.)

Le cardinal de Richelieu est entré dans le panthéon des figures consacrées de la politique internationale notamment à la faveur du culte que lui a voué Henry Kissinger. Les affinités entre ces deux regards d'un même narcissisme froid ont survécu au passage du temps et ces deux personnages sont les archétypes internationaux du rapport entre pouvoir et savoir.

Dans son dernier traité sur la diplomatie, Henry Kissinger retrace en une grande fresque l'histoire de l'art de gouverner le monde, de l'origine de l'âge moderne jusqu'à nos jours ${ }^{5}$. Le XVII ${ }^{\mathrm{e}}$ est un moment fondateur et la guerre de Trente Ans marque le début d'une ère de plusieurs siècles. L'État 
s'impose alors comme acteur central de la politique internationale à la suite de l'Église et des empires. C'est le projet de Henry Kissinger de situer l'émergence de la Realpolitik dans l'équilibre européen issu du traité de Westphalie de 1648. Dans cette généalogie, Richelieu est un précurseur; il est le père originaire de l'équilibre de puissance et le père spirituel d'une Realpolitik européenne ${ }^{6}$.

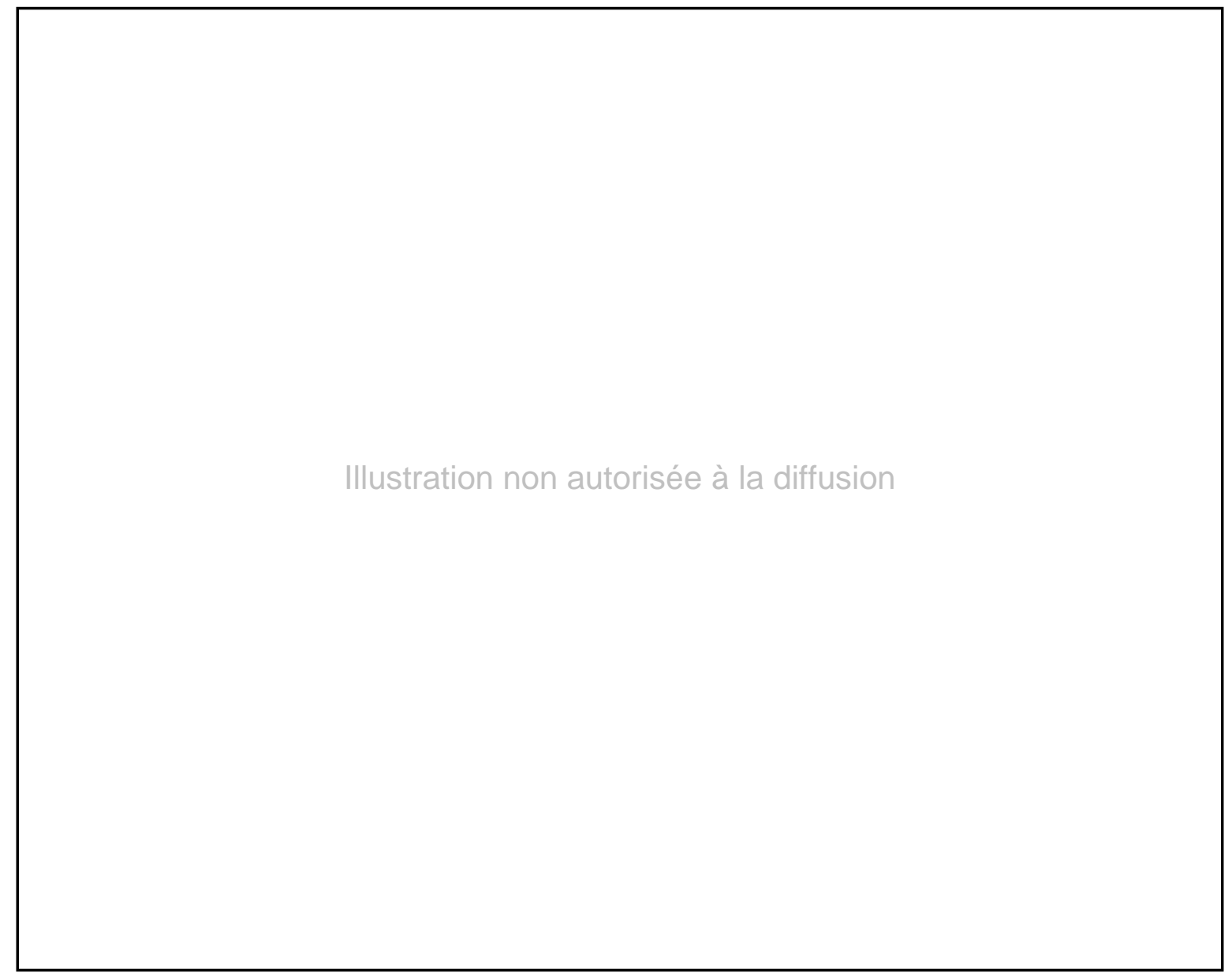

Le portrait du cynisme froid.

(Henry Kissinger. (C) Alen McWeeney/Corbis Outline.)

Le parallèle entre Kissinger et Richelieu est saisissant. L'un comme l'autre, ils sont les auxiliaires du pouvoir en vertu de leur raison présumée, fondée sur une clairvoyance distanciée. Ils incarnent le sens de l'État, la maîtrise de l'information comme sa centralisation au profit d'une stratégie. Ces deux personnages renforcent l'exécutif, qu'il soit royal ou présidentiel. Ces courtisans se distinguent par le vaste crédit dont ils bénéficient auprès du Prince. Auréolées du prestige que confèrent les sommets de la culture légitime - l'Église pour l'une, l'Université pour l'autre -, ces figures sont « censées savoir » parce qu'elles « voient juste».

Leur regard est supposé impartial : pour cela il est considéré comme 


\section{Ariel Colonomos}

infaillible, valorisé par le Prince. Précisément, cette impartialité a été mise à l'épreuve lorsque ces dignitaires ont eu à traiter de questions internationales qui n'étaient pas sans rapport avec leur identité personnelle. Richelieu fit ses preuves et donna des gages de la nature impartiale de sa raison. Il ouvra pour renforcer le catholicisme au sein du royaume tandis qu'il s'employait à diminuer la puissance du Saint Empire romain germanique, nuisant ainsi à l'Église de Rome. Kissinger fut, quant à lui, confronté au conflit israélo-arabe. Son identification à Richelieu aurait pu l'entraîner à faire fi de son identité et à faire preuve de froideur au regard d'une question qui devait être résolue exclusivement à travers le prisme du calcul et de l'intérêt bien compris. La diplomatie kissingérienne au Proche-Orient - shuttle diplomacy - visait en effet à comprendre les intérêts des différents protagonistes de la région pour créer une stabilité favorable, en définitive, aux intérêts des États-Unis. Un tel détachement lui fut reproché en Israël lors de la guerre du Kippour, et des manifestations s'opposèrent à sa venue, l'accusant de favoriser la politique de l'ennemi. Kissinger se départit de sa réserve habituelle : il réagit avec véhémence en déclarant que treize membres de sa famille avaient péri dans les camps de concentration; il lui était, en conséquence, impossible de ne pas se sentir concerné par la survie de l'État israélien. Proclamation de l'émotion qui nous confronte à une alternative : pour l'interpréter, il faut choisir entre la réfutation par Kissinger de sa propre thèse et sa validation par le mensonge dissimulateur.

Cette anecdote est révélatrice de la dimension irréductible de la personne dans la définition d'une posture internationale, aussi bien pour le savant que pour le politique. La dé-personnalisation que le réalisme froid a voulu introduire comme élément fondateur de cette science se révèle, avant tout, être un leurre. Pour survivre, le réalisme doit se mouvoir dans l'ambiguïté ; pour justifier la rigueur de ses thèses, ses apôtres doivent s'abaisser à prétexter que l'émotion qu'ils trahissent est feinte.

\section{L'énigmatique regard des "Ambassadeurs".}

Reprenons ces questions en portant notre attention sur une des ouvres picturales les plus emblématiques de la trajectoire occidentale de la diplomatie. Hans Holbein peint Les Ambassadeurs en 1533 a Londres, où il sera bientôt nommé peintre valet à la Cour. Désireux de décorer son château avec un de ses portraits et admiratif de l'oeuvre d'Holbein, l'ambassadeur de François Ier, Jean de Dinteville, lui commande ce tableau. Son ami, l'évêque de Lavaur George de Selve, s'empresse de rejoindre à Londres Jean de Dinteville pour poser devant Holbein. 
Tout comme les portraits de Richelieu, cette ouvre pose la question de l'identité à une époque profondément marquée par les guerres de Religion. Les protagonistes de cette mise en scène de la puissance affichent pourtant des signes impersonnels de reconnaissance, leur identité en tant que personnes est cachée sous leur impassibilité. Tout renvoie chez eux à un marquage de l'identité par le social. Ces représentants du pouvoir font avant tout ostentation de leur origine sans que pour autant les marques de reconnaissance sociale nous renseignent sur leurs opinions, leurs sentiments, leurs émotions. Les deux diplomates sont essentiellement des hommes d'État, dans cette stature il n'y a guère de place pour l'identité personnelle. L'un est le fils d'un ami de François ${ }^{\text {er }}$ et est richement vêtu; l'autre est évêque, ses atours sont sobres, mais son manteau est là pour indiquer un rang élevé. Leur jeune âge est un autre gage de puissance. Dans le dressage des corps et l'attribution des fonctions, la tradition est à peu près respectée - en effet, on rappelle dans les manuels de diplomatie que le "parfait ambassadeur ${ }^{7}$ " ne doit pas avoir moins de trente ans ni plus de cinquante ${ }^{8}$.

Cette démonstration de force, de puissance et de richesse s'accompagne, comme dans le cas de Richelieu, d'une impassibilité du regard et d'une impénétrabilité des traits. Certains commentateurs de ce tableau ont cru déceler l'expression de l'ennui chez Dinteville, un ennui emblématique de la diplomatie, tandis que l'évêque de Lavaur paraît trahir une certaine inquiétude 9 .

Le regard que Les Ambassadeurs portent sur le monde n'est pas uniquement lisible dans les yeux des personnages. Les objets occupent dans ce tableau une place essentielle, leur ostentation traduit un intérêt notable pour la matérialité et le luxe de la Renaissance ${ }^{10}$. Ces choses sont porteuses de sens : les objets disposés sur la table qui à la fois sépare et unit les deux hommes en permettant de les placer l'un par rapport à l'autre de manière symétrique sont de précieux instruments des sciences et des arts.

Ce sont précisément des objets du voir. La science de la vision est à la disposition de l'homme d'État. Sur l'étage supérieur de la table sont représentés des objets de mesure scientifique qui procurent à l'homme la sensation de maîtriser l'espace : il s'agit d'un sextant, d'un cadran solaire, d'un astre céleste, posés sur une table recouverte d'un précieux tissu aux dessins géométriques. L'astre céleste comme le cadran solaire sont à la hauteur des yeux des deux personnages. Leur regard a pour ambition de saisir le monde et d'appréhender la mesure de sa réalité; ce regard froid, impassible et distancié veut faire œuvre de science. On retrouve ici les trois versants de notre exploration sur le regard : les figures du diplomate, du peintre et du scientifique cohabitent sur la scène de ce tableau. Le regard impassible du diplomate est peint par un artiste qui figure l'attitude 
Hans Holbein, Les Ambassadeurs, 1533.

(C Bridgeman-Giraudon.)

figée des représentants de l'État à travers leur identification aux sciences de l'espace. Ce réalisme en peinture associe le diplomate à des instruments de mesure de la réalité qui sont là pour indiquer l'émergence d'une science de la maîtrise du monde.

Celle-ci préfigure l'interprétation réaliste comme science rationnelle de l'international. Bien plus tard, au milieu du $\mathrm{XX}^{\mathrm{e}}$ siècle, le réalisme se développera en puisant son inspiration dans certains grands textes fondateurs de la politique dont les auteurs ont valorisé l'insensibilité et le calcul : Thucydide, Machiavel, Hobbes, Clausewitz. Cette analyse est fondée sur une opération dont les termes sont potentiellement contradictoires l'un par rapport à l'autre : voir en étant insensible à ce qui détourne le regard de la réalité. Ainsi définie, une telle vision procède de l'aveuglement. Nous le verrons, cet aveuglement est le symptôme d'un refoulement. 
Le tableau d'Holbein pose la trame d'une réflexion décisive sur la nature de la politique internationale. L'ambassadeur et l'évêque son complice - qui a été précédemment l'envoyé de François I ${ }^{\text {er }}$ auprès du Saint-Siège - se cachent derrière leur impassibilité pour éviter de trahir des sentiments qui pourraient être interprétés à leur détriment par leurs adversaires. Par ce stratagème, le politique use de la puissance pour prévenir les effets de sa hantise de l'impuissance. Le diplomate doit faire preuve de retenue dans son expression et fait montre de sa force pour éviter de dévoiler sa faiblesse. À moins qu'elle ne soit feinte, l'émotion est une marque de faiblesse qu'il faut masquer. Dinteville occupe une place majestueuse dans cette peinture : l'œuvre sert de renfort narcissique à son commanditaire, de bouclier contre les aspérités du réel. Par ailleurs, ce tableau vient enrichir la collection de Dinteville, qui déjà possède un portrait de lui-même où il figure en saint Georges : à ses pieds gît la tête du dragon qu'il vient de terrasser ".

La froideur est une dissimulation stratégique, à peine voile-t-elle le désir de jouer avec le regard de l'autre ${ }^{12}$. En faisant montre d'une force déterminée par la raison, le diplomate - un bloc de certitudes - influence le regard de celui auquel cette image est destinée. La neutralité du visage tout comme la preuve objective de la force sont autant de signaux destinés à valoriser l'homme d'État et le diplomate en orientant le regard de celui qui les observe, au sein même de la Cour ou chez les dirigeants des autres nations. Cet art de la parade et de la communication procède, en règle générale, d'une intuition bien avisée.

La théorie des relations internationales fait écho à cette représentation du pouvoir. À l'âge thermonucléaire, ce face-à-face des regards a été analysé par Robert Jervis, qui a saisi toute l'importance de la communication sur la scène internationale ${ }^{13}:$ il a indiqué le rôle des signaux, des messages visant à influencer la perception de soi du destinataire de ces adresses.

De ce point de vue, l'œuvre d'Holbein constitue un double signal. D'une part, elle a une fonction à la Cour, où elle est censée grandir Dinteville et Selve; d'autre part, elle a vocation de stratégie en soulignant la fonction stabilisatrice de la France dans les guerres de Religion. Les Ambassadeurs suggèrent la gravité des troubles entre catholiques et protestants. Forts de cette dramatisation, ils affirment le rôle des diplomates laïcs et religieux dans le gouvernement des âmes.

\section{La déraison du monstre froid.}

Le tableau d'Holbein signifie le désir d'exhiber une identité sociale, de faire ouvre de monstration. Il donne à voir l'impersonnel. Ce faisant, il pose, par l'énigme, la question de l'humain. L'anamorphose de crâne est 
presque dissimulée, il faut pencher son regard pour prendre véritablement la mesure de ce Memento mori («Souviens-toi de la mort " était la devise de Dinteville ${ }^{14}$ ). Un tel voilement fait le propre de l'œuvre. L'anamorphose qui siège aux pieds des deux dignitaires introduit une séparation dramatique. Cette division rappelle la force du principe de symétrie - le politique vis-à-vis du religieux, comme la science vis-à-vis des arts - qui fait écho à une division plus essentielle, entre la vie et la mort.

S'inspirant de la tradition des vanités, Holbein place la mort au centre de son œuvre. Le jeu entre le travail de la puissance des Ambassadeurs et le caractère irréductible de la mort est intéressant à plus d'un titre. En premier lieu, il indique une représentation de la puissance dont la mort est l'implicite substance : l'État doit faire montre de puissance pour susciter un effet de dissuasion, afin d'éviter qu'un ennemi n'ait le projet d'envahir son territoire. La puissance est donc nécessairement liée à l'impuissance, à travers l'éventualité de la mise à mort dont il faut se protéger en l'anticipant. Cette vision de la politique procède de la métaphore du jeu à somme nulle : la survie passe par la mort de l'autre.

La mise en scène de la puissance par la peinture pose une autre question décisive. Le regard impassible des protagonistes de cette scène de cour signifie leur capacité à maîtriser leurs sentiments, la force de leur retenue, qualités indispensables à l'exercice de l'art de gouverner et de négocier. Cette retenue est la métaphore d'une psychologie, elle traduit un caractère déterminé et résolu.

Le Memento mori nous le rappelle, la retenue est la façade de la détermination, l'insensibilité, un bouclier. Dans de telles conditions, la civilité présagerait la barbarie. La tradition réaliste qui érige en principes la maximisation de la puissance et la défense de l'intérêt national puise son inspiration dans des philosophies qui, chacune, suggèrent au gouvernant de faire montre de froideur et d'insensibilité dans l'exercice de son pouvoir. À ce titre, Les Ambassadeurs font écho à une règle élémentaire : plus l'homme d'Etat et le militaire seront en mesure de faire preuve de retenue dans l'expression de leurs sentiments et de leurs émotions, plus ils seront à même de prendre les décisions nécessaires à la mise en acte de la force. La retenue psychologique et verbale s'accompagnerait d'une absence de retenue dans la violence physique légitime; le sang-froid rendrait possible la violence extrême. Aussi, Thucydide, Machiavel, Hobbes et Clausewitz incitent le Prince non seulement à exhiber sa force pour dissuader des ennemis ou des rebelles de l'inquiéter, mais également, lorsqu'ils le jugent opportun, à prendre l'initiative d'une violence sans discrimination ni limites. Est ici clairement affirmée la justification de l'usage extrême de la force. La tradition clausewitzienne a, dans ce domaine, marqué les esprits et on se souvient du principe établi par le général prussien : 
Les âmes philanthropiques pourraient alors aisément s'imaginer qu'il y a une façon artificielle de désarmer et de battre l'adversaire sans trop verser de sang, et que c'est à cela que tend l'art véritable de la guerre. Si souhaitable que cela puisse paraître, c'est une erreur qu'il faut éliminer. Dans une affaire aussi dangereuse que l'art de la guerre, les erreurs dues à la bonté d'âme sont précisément la pire des choses ${ }^{15}$.

Il n'en demeure pas moins qu'Holbein, quant à lui, est un humaniste, un admirateur d'Érasme, qui a été son protecteur ${ }^{16}$. Les Ambassadeurs traduisent certaines des préoccupations de l'humanisme de la Renaissance et, à travers le thème des vanités, Holbein se fait le critique du pouvoir dans son association avec une science de la guerre. Une telle critique se fonde sur une métaphysique. En considérant la mort, le peintre montre que la froideur de la maîtrise, de l'anticipation et de la retenue toujours suppose son inverse : l'imprévisible dérèglement de la déraison.

Sur le plan politique, ces considérations ont un fondement mécaniste et fonctionnaliste. La diplomatie est une tentative de régler par la paix les différends des États, elle est un antidote à un mal dont elle veut se prémunir en conjurant ses effets les plus mortifères. Pour autant, la diplomatie vit de la guerre qu'elle veut éviter. À ce titre, la peinture d'Holbein introduit une réflexion décisive sur la violence et la retenue dans les affaires de l'État en indiquant une tension profonde entre la froideur du calcul qui doit conjurer la mort par la puissance et la certitude refoulée de la finitude. La mort, en effet, est la division ultime qui donne tout son sens au tableau. Plus les grands cherchent un abri dans l'illusion de la maîtrise du monde, plus ils sont exposés à leur condition mortelle. Ni les sciences ni les arts ne détourneront l'ambassadeur et l'évêque de leur destination finale. Le contraste est insupportable entre leur impassibilité et l'anamorphose qui représente le refoulé de la puissance calculatrice. Tout en étant certaine, la mort est ce qui ne suppose aucune anticipation, l'imprévisible.

Elle est à l'image de la disparition dans des conditions tragiques des protagonistes de cette scène de cour. Quelques années après avoir posé pour Holbein, Dinteville fut atteint d'une paralysie qui figea les dix dernières années de sa vie ${ }^{17}$. Le sort de Selve fut aussi funeste : il décéda prématurément à l'âge de trente-trois ans. Quant à Holbein (son nom veut dire « os creux » en allemand), la mort a hanté sa vie, précisément dans ses rapports avec un être cher, véritable prolongement de lui-même. À travers la représentation du moi et du double, Les Ambassadeurs nous renvoient, en effet, à un tragique épisode de la vie d'Holbein. Son frère aîné Ambrosius était peintre également et ils avaient réalisé ensemble de nombreux tableaux. Or Ambrosius mourut en 1519, à l'âge de vingt-trois ans ${ }^{18}$. La propre fin d'Holbein fut elle aussi marquée par le tragique : sept ans après avoir été nommé peintre à la cour d'Angleterre, en 1543, il succomba à la peste qui s'abattit sur Londres. 
La froideur signifie la prévision, elle ne fait que tenter - en vain - de conjurer l'imprévisibilité de la séparation et de la disparition. Si l'on considère le caractère imprévisible de la mort, la stratégie devient illusoire et la rationalité irrationnelle. Un tel scepticisme sur le plan ontologique rejoint des considérations contemporaines sur la stratégie militaire. Dans ce domaine, le thème de l' " irrationalité de la rationalité » a inspiré certaines études critiques de la stratégie pendant la guerre froide. Alors que le réalisme s'impose comme le modèle dominant d'une science prônée par des théoriciens et des courtisans qui tentent, par leur regard infaillible, de séduire les grands, une préoccupation majeure hante l'ère du nucléaire : la définition du meilleur calcul stratégique dans un univers où une simple erreur peut mettre en danger toute la planète (MAD ${ }^{19}$ ).

Dans le registre des représentations, une nouvelle figure est ici pleinement éclairante. Le Dr. Strangelove incarné par Peter Sellers dans le film de Stanley Kubrick est sans doute l'image qui a le mieux illustré l'antinomie retenue/déraison. Strangelove est l'énigmatique stratège qui a vocation à conseiller le président des États-Unis à l'occasion d'une crise nucléaire d'une intensité dramatique. L'heure est grave: une erreur humaine a déclenché un processus irréversible de mise à feu des missiles américains, qui se dirigent vers leurs cibles soviétiques. Les mouvements de Strangelove sont raides et saccadés, ce personnage est un sombre pantin qui se déplace dans un fauteuil roulant. Aussi, cette retenue maximale s'accompagne de crises sur un mode hystérique au cours desquelles il perd le contrôle de luimême. Son regard est voilé de noir, il est recouvert afin de cacher ce qui transparaît derrière sa tentative de contenir ses pulsions meurtrières.

Les scènes ultimes de ce récit de l'État qui perd sa raison sont consacrées à un savant calcul eugéniste. Quelles personnes pourraient être sauvées et se cacher sous la surface de la terre en attendant que les effets de la radioactivité se dissipent. Strangelove propose de sélectionner les hommes dont les qualités les rendraient les mieux à même de perpétuer l'espèce, en l'occurrence les élites de l'État. Ils seraient accompagnés d'un nombre supérieur de femmes pour que le temps passé dans ce souterrain ne leur paraisse pas trop fastidieux. Le séjour de ces nouvelles générations de subterriens devra en effet durer près d'un siècle - c'est le résultat de l'estimation de Strangelove qui évalue le temps de la protection de la mort par radiation. Soudain, le visage du stratège s'éclaire d'un sourire, son bras inerte est secoué par un tremblement et se hisse dans un salut nazi, son verbe échappe à son contrôle. Le stratège incarne dans ce rôle la figure de la déraison, l'illusoire maîtrise par la prévision et le calcul.

La déraison est la face sombre d'une telle rationalité. La froideur de l'insensibilité est le propre d'une monstruosité qui se révèle dans la crise. 
L'État, ce monstre froid, est un Janus. L'insensibilité de la froideur est opératoire dans le langage de la guerre tandis que dans les situations extrêmes transparaît, sous la forme de l'exutoire, une inquiétante jouissance.

L'État compose des scènes tragiques - la dissuasion, la montée aux extrêmes, la crise à proprement parler - en espérant que la raison le pro- 


\title{
Ariel Colonomos
}

tège de la défaillance. In fine, la raison, le calcul et les intérêts sont autant de justificatifs de la disposition des représentants de l'État à se dédouaner de la critique rédhibitoire qui les accuserait de céder à leurs passions ${ }^{21}$. Les grands croient se protéger en posant un regard qui soutient un refoulement entendu comme maîtrise de la maîtrise. Pour l'homme, c'est là une retenue pour le moins ambiguë : une maîtrise de ses émotions qui signifie la volonté de puissance et une absence de retenue dans la violence physique qui s'accompagne du refoulement de sa propre mort.

\author{
Ariel Colonomos \\ colonomos@ceri-sciences-po.org \\ CNRS-CERI
}

\section{NOTES}

1. «De la nouvelle idole », dans Ainsi parlait Zarathoustra (nous soulignons). Stanley Hoffmann a repris ce terme dans un de ses ouvrages (Une morale pour les monstres froids), contribuant ainsi à son adoption dans l'ensemble d'une discipline.

2. Des sens à la raison, on retrouve la direction kantienne. "Toute notre connaissance commence par les sens, passe de là à l'entendement et s'achève dans la raison, au-dessus de laquelle il n'y a rien en nous de plus élevé pour élaborer la matière de l'intuition " (Kant, Critique de la raison pure, Paris, PUF, 1968, p. 254).

3. Voir Hans Morgenthau, Politics among Nations: The Struggle for Power and Peace. La célèbre théorie réaliste de la guerre, telle qu'elle est notamment établie dans cet ouvrage, l'un de ses plus classiques, suppose un versant explicatif ainsi qu'une dimension normative. Le réalisme vise à la fois à expliquer le comportement des acteurs internationaux (les Etats) et à suggérer à leurs représentants la meilleure des conduites des affaires de la guerre et de la paix. Au nom de cette théorie qui fait sa promotion en doctrine, ou à l'inverse de cette doctrine qui prend la parole au nom d'une théorie, les intérêts des États dictent leur agir sur l'échiquier international. On veut ainsi tirer de ce que l'on croit observer dans la réalité les règles de ce que l'on devrait faire.

4. L'historien décèle ce trait et l'analyse de manière très convaincante dans son travail sur la " fabrication » de Louis XIV; voir Peter Burke, Louis XIV. Les stratégies de la gloire.

5. Henry Kissinger, Diplomacy.

6. Voir ibid., p. 56.

7. Voir Garrett Mattingly, Renaissance Diplomacy, p. 155; et Don Juan Antonio de Vera, Le Parfait Ambassadeur.

8. Lorsque Holbein peint Les Ambassadeurs, Dinteville a vingt-neuf ans.

9. Susan Foister, Ashok Roy et Martyn Wyld, Making and Meaning: Holbein's "Ambassadors".

10. Voir Lisa Jardine, Wordly Goods. A New History of the Renaissance.

11. Francesco Primaticcio, Jean de Dinteville as Saint George (c. 1544/1545), reproduit in S. Foister, A. Roy et M. Wyld, Making and Meaning, p. 23.

12. Dans une partie de son séminaire consacré au regard, Lacan avait judicieusement noté à propos des Ambassadeurs : "Ce tableau n'est rien d'autre que ce que tout tableau est, un piège à regard " (Le Séminaire, livre XI, Les Quatre Concepts fondamentaux de la psychanalyse, p. 83). 


\section{La froideur du regard impassible des Etats}

13. Pour une interprétation d'inspiration goffmanienne des jeux internationaux, voir Robert Jervis, The Logic of Images in International Relations et Perception and Misperception in International Politics.

14. Voir Jean-Louis Ferrier, Holbein - «Les Ambassadeurs », p. 29.

15. Carl von Clausewitz, De la guerre, première partie, livre I, "La nature de la guerre ", $\S 3$, «Usage illimité de la force », p. 52.

16. Voir Jurgis Baltrusaïtis, Anamorphoses ou Magie artificielle des effets merveilleux, p. 91 sq.

17. Voir Jean-Louis Ferrier, Holbein - «Les Ambassadeurs », p. 9.

18. Ibid., p. 30. Ambrosius et Hans Holbein avaient peint un diptyque qui représente deux enfants : Ambrosius avait réalisé les visages et Hans avait peint des crânes d'adultes.

19. Mutual Assured Destruction.

20. Dr. Strangelove or How I Learned to Stop Worrying about the Bomb (1963), film de Stanley Kubrick tiré du roman de Peter George, Red Alert.

21. Un tel phénomène a été très clairement analysé par Freud : «ils [les peuples] mettent en avant leurs intérêts pour pouvoir trouver les raisons à la satisfaction de leurs passions" ( "Considérations actuelles sur la guerre et sur la mort ", p. 25).

\section{BIBLIOGRAPHIE}

AGRIPPA, Henry Corneille, De l'incertitude. Vanité et abus des sciences, traduit en françois par Louys de Mayerne Turquet Lyonnois, imprimé en l'an de grâce MDCXXX.

BALTRLSAIITIS, Jurgis, Anamorphoses ou Magie artificielle des effets merveilleux, Paris, Olivier Perrin, 1969.

BELY, Lucien, Espions et Ambassadeurs au temps de Louis XIV, Paris, Fayard, 1990.

BELY, Lucien (dir.), L'Invention de la diplomatie. Moyen Age-Temps modernes, Paris, PUF, 1998.

BURKe, Peter, Louis XIV. Les strategies de la gloire (New Haven, Yale University Press, 1992), Paris, Éd. du Seuil, 1995.

Clausewitz, Carl von, De la guerre, Paris, Minuit, 1955.

Courtine, Jean-Jacques, et HAROCHE, Claudine, Histoire du visage. Exprimer et taire ses émotions $\left(X V I^{e}\right.$-début $\left.X I X^{e}\right)$, Paris, Payot, 1994.

EI.IAS, Norbert, La Société de cour, Paris, Flammarion, 1985.

FERrier, Jean-Louis, Holbein - "Les Ambassadeurs ", Paris, Denoël, 1977.

FolsTER, Susan, ROY, Ashok, et WYLD, Martin, Making and Meaning Holbein's "Ambassadors ", Londres, National Gallery Press, 1997.

Freud, Sigmund, “Considérations actuelles sur la guerre et sur la mort " (1915), in Essais de. psychanalyse, Paris, Payot, 1981, p. 7-40.

Hoffmann, Stanley, Une morale pour les monstres froids. Pour une ethique des relations internationales, Paris, Ed. du Seuil, 1982.

JaRDine, Lisa, Wordly Goods. A New History of the Renaissance, Londres, Macmillan, 1996.

JERVIS, Robert, The Logic of Images in International Relations, Princeton University Press, 1970.

- Perception and Misperception in International Politics, Princeton University Press, 1976.

KISSINGER, Henry, Diplomacy, New York, Simon and Shuster, 1994.

LACAN, Jacques, Le Séminaire, livre XI, Les Quatre Concepts fondamentaux de la psychanalyse, Paris, Éd. du Seuil, 1973.

LEUSSE, Meredith Kingston de, Diplomate. Une sociologie des ambassadeurs, Paris, L'Harmattan, 1998.

Matringl,y, Garrett, Renaissance Diplomacy (1955), New York, Dover Publications, 1988. 


\section{Ariel Colonomos}

Morgenthal, Hans, Politics among Nations: The Struggle for Power and Peace, New York, Knopf, 1985 (1 $1^{\text {re }}$ éd. New York, McGraw Hill, 1948).

NiCOLSON, Harold, Diplomacy, Londres, Oxford University Press, 1963.

Selve, George de, Euvres de Feu Révérend Père en Dieu, Contenans un sermon... Moyen de faire et entretenir la paix, deux Remontrances aux Allemans, Paris, 1559.

STOICHITA, Victor, L'Instauration du tableau, Paris, Droz, 1999.

VerA, Don Juan Antonio de, Le Parfait Ambassadeur (1620), trad. fr. 1642.

WIGHT, Martin, International Theory The Three Traditions, Londres, Royal Institute of Affairs, 1996.

\section{RESUME}

À travers l'analyse de certains portraits de grands dignitaires de l'État, ce texte explore les rapports entre une esthétique de la représentation du pouvoir et une rationalité d'une science réaliste des relations internationales fondée sur la distanciation amorale. Dans l'incarnation de l’État par les grands, cette esthétique du regard communique avec cette science de la vision.

Notre analyse montre que le trait précède la science et qu'à travers le travail du peintre l'intuition du regard de l'État préfigure la construction d'un savoir froid et objectivant. Les correspondances entre ces trois regards - le peintre, le diplomate et le savant - sont à l'origine d'une énigme dont la raison du politique est le cœur.

\section{SUMMARY}

The esthetics of power have anticipated and influenced the construction of a rational and amoral theory of international relations called realism. In this paper, the analysis of several portraits of statesmen and diplomats focuses on the representation of the eyes of power. Three major characters have to be taken into account in this process: the painter, the diplomat and the scholar. They are the true protagonists of the emergence of this rationality that has played a great role, for better and most often for worse, in international politics. 